\title{
The Laser Alignment System of the CMS Silicon Strip Tracker
}

\author{
Jan Olzem* \\ DESY Hamburg \\ on behalf of the CMS Collaboration \\ E-mail: jan.olzem@cern.ch
}

The Laser Alignment System (LAS) of the CMS Silicon Strip Tracker has been designed for surveying the geometry of the large-scale tracker support structures. It uses 40 laser beams (wavelength $\lambda=1075 \mathrm{~nm}$ ) that induce signals on a subset of the tracker silicon sensors. The spatial resolution of the reconstructed laser spots is $30 \mu \mathrm{m}$.

This allows the LAS to continuously monitor on time the position of the different tracker components relative to each other with a precision better than $30 \mu \mathrm{m}$. Additionally, it can provide an absolute measurement of the tracker mechanical structure with an accuracy better than $70 \mu \mathrm{m}$, thereby supplying additional input to the track based alignment at detector startup.

31 out of the 40 LAS beams have been successfully operated during the CMS cosmic muon data taking in autumn 2008. The alignment of the tracker end cap disks and of the disks with respect to the Tracker Inner Barrel and Tracker Outer Barrel subdetectors was measured with a precision of $70 \mu \mathrm{m}$. With the operation of the full set of LAS beams in summer 2009, even higher precision is expected. This new measurement will provide the reference for the monitoring of the stability of the tracker mechanical structure during physics runs with an accuracy better than $30 \mu \mathrm{m}$.

9th International Conference on Large Scale Applications and Radiation Hardness of Semiconductor Detectors, RD09

September 30-October 2, 2009

Florence,Italy

\footnotetext{
* Speaker.
} 


\section{The CMS Silicon Strip Tracker}

The CMS Silicon Strip Tracker is the largest silicon microstrip detector ever built with a sensitive area of about $200 \mathrm{~m}^{2}$. It is divided into an inner barrel/end cap structure, Tracker Inner Barrel (TIB) and Tracker Inner Disks (TID), and an outer structure consisting of an outer barrel (TOB) and two end caps (TEC). Its overall dimensions are $5.6 \mathrm{~m}$ length and $2.4 \mathrm{~m}$ diameter (Fig. 1). The basic elements of the Silicon Strip Tracker are the modules, each of which carries one or two sensors and the front-end electronics. In TIB and TOB, the modules are arranged in four and six cylindrical layers, respectively, on longitudinal structures parallel to the beam mounted on the layer half-barrels. TID modules are grouped in three disk units per side on ring-shaped concentrical structures. In the TEC, modules are placed in ring structures on wedge shaped "petals" which are themselves attached on both sides to nine large disks staggered along z. A detailed description of the Silicon Tracker can be found in [1].

The CMS coordinate system has the origin centered at the nominal collision point inside the experiment. The $y$-axis is pointing vertically upward, and the $x$-axis radially inward toward the center of the accelerator. The $z$-axis points along the beam direction, completing the right-handed coordinate system.

\section{The Laser Alignment System}

The Silicon Strip Tracker is operating at $-20^{\circ} \mathrm{C}$ and will undergo several temperature cycles during its lifetime, which impose mechanical stress on the carbon fiber support structure. Additionally, possible changes in humidity may result in a deformation of the tracker support material. The task of the hardware based laser alignment system (LAS) is the initial absolute measurement of the large-scale support structure of the Silicon Strip Tracker with an accuracy below $70 \mu \mathrm{m}$, and its continuous on-time monitoring relative to a previous measurement with better than $30 \mu \mathrm{m}$ precision.

\subsection{Working principle and layout}

The LAS uses a system of near-infrared laser beams $(\lambda=1075 \mathrm{~nm})$ which traverses the silicon in the tracker volume (Fig. 1). By inducing signals on the silicon sensors, they serve as a reference coordinate system for the inter-alignment of the barrel subdetectors and the end cap disks. The LAS requires no additional sensors and makes use of the existing data acquisition system of the tracker. Single laser hits can be reconstructed with a spatial resolution of about $30 \mu \mathrm{m}$. The LAS has a large sensitive range for residual measurements up to several millimeters; furthermore, it provides very fast response, so that full measurement and reconstruction can be performed on the time scale of a minute. The inner disks and the pixel detector are not surveyed by the LAS.

The laser beams are eight-fold distributed along the $\phi$ coordinate of the tracker and divided into two groups:

- eight "alignment tube" beams range from one end cap to the other end cap and interconnect TIB, TOB and both TECs at a radius of $56.4 \mathrm{~cm}$ from the beam line; 
- two separate systems of end cap internal beams provide inter-alignment of the TEC disks. In each TEC, a total of 16 beams traverse all silicon layers at radii of $56.4 \mathrm{~cm}$ and $84.0 \mathrm{~cm}$, respectively.

In the end caps, three of the alignment tube beams overlap with the end cap internal beams at the smaller radius, such that the respective modules are hit by two beams at the same nominal positions.

Light is distributed in the tracker volume via optical fibers, each of which is connected to a beam splitter. For the end cap internal beam system, beam splitters are mounted in the support wheel of TEC disk 6, between the sensors of disks five and six. The alignment tube beam splitters are located between the plus side end cap and the barrel subdetectors.

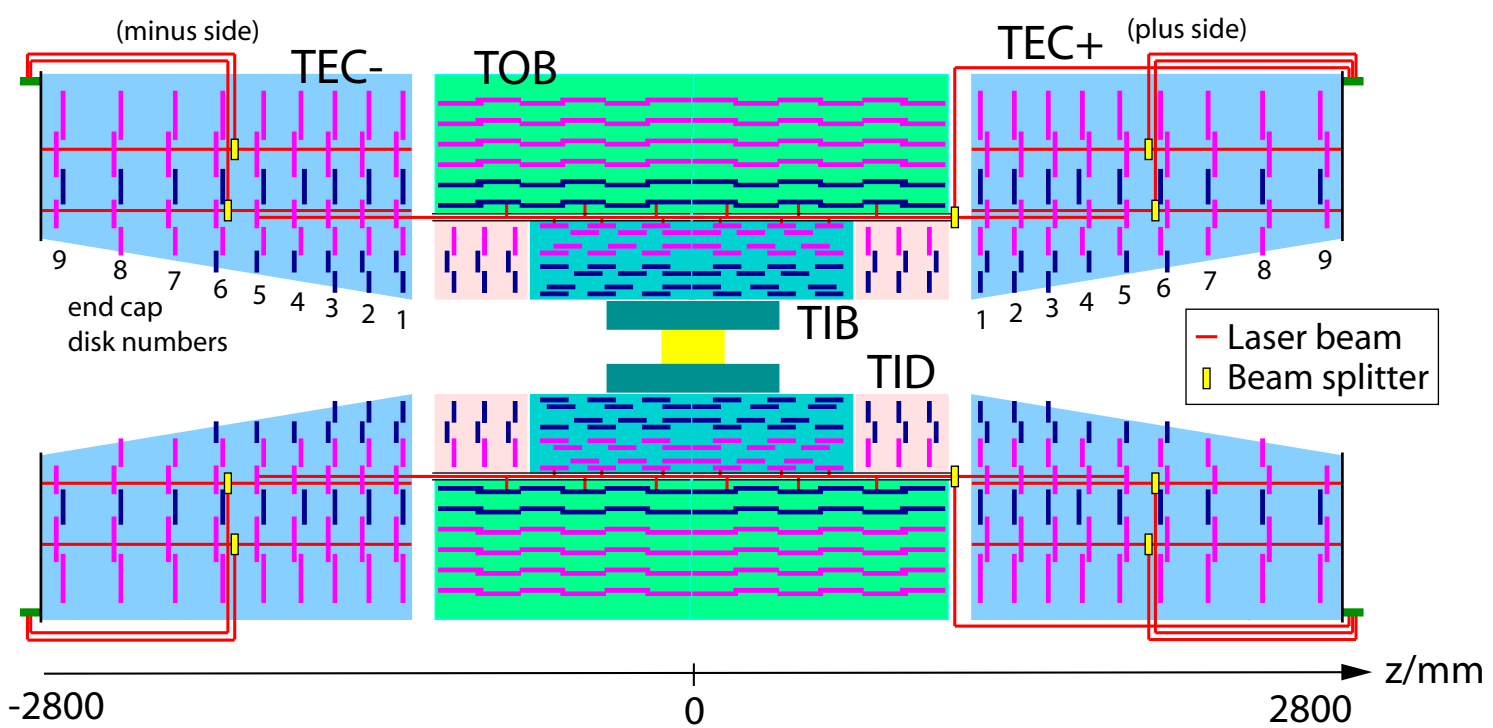

Figure 1: Longitudinal cross section ( $r z$ view) of the CMS Silicon Strip Tracker with the LAS components.

\section{Components}

In the end caps, the laser beams traverse up to 4 consecutive silicon sensors which are by default unpolished and coated with an aluminum layer on the back side. To minimize the reflection and scattering of the light while it traverses the sensors, the sensors hit by the lasers in the end caps underwent a special manufacturing process: 1) a hole with a diameter of $10 \mathrm{~mm}$ was left out in the backplane metallization; 2) the sensor back sides have been polished in this region, and an anti-reflective coating has been applied. With this treatment, a transmission efficiency of 20-30\% can be achieved, while the reflectivity decreases to about $10 \%$.

The beam splitters are connected to the laser diodes via long optical fibers. Using a collimator lens and a prism doublet in conjunction with a $\lambda / 4$ plate and a mirror, they generate a pair of back-to-back beams in the tracker volume. Due to manufacturing tolerances, small deviations from collinearity of these beam arms of the order of $1 \mathrm{mrad}$ are observed. These deviations have been measured before the assembly of the tracker and are corrected for during offline alignment parameter reconstruction. 
As a consequence of polarization conservation in the fibers, the intensity of the two beam arms may differ significantly, depending on the orientation of the fiber segments along their paths. This leads to different signal amplitudes in the silicon sensors on either side of the beam splitter. The effect is compensated during offline reconstruction by taking into account an increased number of laser spot signals from sensors with low amplitudes. It is foreseen to eliminate it with the installation of depolarizing devices in the fiber path close to the diodes.

All beam splitters are mounted on support structure elements of the tracker whose geometry should be measured by the LAS. Therefore, the position and orientation of the beams are not known precisely due to a possible misalignment. In the most general case, the latter must also be assumed to vary with time. This is considered during offline reconstruction of the alignment parameters $(\S 5.2)$.

In the alignment tube beam system, the light is guided through aluminum tubes from one end cap to the other in the space between TIB and TOB ("alignment tubes"). There are two tubes per beam, one on the negative and one on the positive $z$ side. One beam splitter is attached to the outer end of each positive $z$ side tube. Light is deflected to six TIB and six TOB modules per beam by semitransparent mirrors with a reflectivity of about $5 \%$. The coplanarity of the mirrors has been measured prior to the tube assembly and is used for correction during offline processing of the LAS data.

The LAS electronics are located in the service cavern of the CMS experiment. The 40 laser diodes are distributed over five VME boards which provide a programmable logic for defining the width, intensity and delay individually for each laser pulse. Additionally, one VME board decodes the incoming trigger signals and triggers all laser boards upon arrival of a special calibration trigger (TTC-B). The front-end driver electronics of the tracker modules also recognize the TTC-B and route the event data to a specific calibration data stream from which it is picked up for offline reconstruction.

\section{Operation and commissioning}

\subsection{Operation}

The laser VME boards are programmable to deliver a series of up to 3200 consecutive laser pulses, each with individually defined pulse shape and delay settings. Due to the limited transmissivity of the silicon and dynamic range of the front-end electronics, it is not possible to induce appropriate signals on all end cap disks with one single laser amplitude. Thus, the lasers are operated with a repeated sequence of up to five pulses with different amplitude settings, each being individually adapted for particular disks. As a consequence, most modules will be frequently illuminated with insufficient or excessive laser intensity, leading to either a missing signal or to the overload of the front-end electronics, respectively. Due to the overlap of several beams in the end caps ( $\$ 2.1)$, the alignment tube and end cap internal beam systems are operated in alternation. These effects are taken into account during offline data analysis ( $\$ 5.1)$.

During the CMS cosmic muon data taking campaigns in 2008 and 2009, the LAS has been operated in parallel to physics data taking with a calibration trigger rate of $100 \mathrm{~Hz}$. For future operation it is foreseen to trigger the lasers during the LHC abort gaps - where no collisions occur - to avoid any interference with collision data. 
To achieve a signal-over-noise ratio of about 10, 200 laser signals must be accumulated per module. Taking into account the different amplitudes and the altenative operation of the two beam systems, a total number of 2000 laser events have to be collected for a full alignment measurement. Given calibration trigger rates on the order of $100 \mathrm{~Hz}$ as stated above, data for such a measurement can be acquired within a few seconds.

\subsection{Commissioning: timing and intensity scans}

Operation of the LAS during the CMS cosmic muon data taking was mainly devoted to commissioning of the system. In the first step, the correct timing settings were determined. The trigger VME board offers the possibility to delay the laser pulses globally with respect to the incoming calibration trigger in units of bunch crossings ( $25 \mathrm{~ns}$ ). Additionally, each laser pulse can be aligned in time with a programmable delay in units of nanoseconds. In this manner, the timing can be corrected for the front-end electronics latency, the global calibration trigger delay, as well as for the fiber lengths and the photon time of flight.

Moreover by scanning over a range of laser intensities, a set of operation parameters for the laser diodes was found which allows to induce good signals on all end cap layers.

\section{Offline reconstruction}

\subsection{Beam spot reconstruction}

The beam spot positions are reconstructed from the analysis of the signals of the 512 strips on each module. Fig. 2 shows two examples of such event "profiles" for data taken in raw and zero-suppressed ${ }^{1}$ mode. As a consequence of the diffraction in the strip metallization, several side peaks can be observed in the profiles. The number of peaks depends on the number of sensors traversed by the beam on the way from the beam splitter to the module. The peak of zeroth order has almost a Gaussian shape with a slight deviation due to non-ideal beam optics and interference from multiple reflections inside the silicon.

The left panel of Fig. 2 displays the effect of excessive laser amplitude on the front-end electronics of a module, which becomes visible in raw data taking mode. The high current induced by the laser pulse decreases the effective pedestals via the built in common mode noise correction circuitry, such that - after nominal pedestal subtraction - the resulting response from the affected channels appears to have a negative amplitude. In some cases, the slope in the baseline may result as a shift of the peak position, thus a set of filter algorithms are deployed to recognize such profiles and reject them before further analysis.

On the right hand side of Fig. 2, a profile from data taken in zero-suppressed mode is shown. In this mode, effects from excessive laser amplitude are hidden by the suppression algorithm, so that the filter algorithms must be replaced by a tight cut on the maximum peak amplitude. Finally, for the beam spot reconstruction, the peak of zeroth order is identified and its position is determined by fitting a Gaussian function. The precision of the beam spot reconstruction is mostly limited by the systematic deviations of the peaks from their Gaussian shape. The combined statistical and systematic error has been determined using simulated data and is about $30 \mu \mathrm{m}$.

\footnotetext{
${ }^{1}$ In the zero-suppressed mode, the front-end electronics transfer only signals from channels with a signal-over-noise above threshold (nominal physics data taking mode).
} 

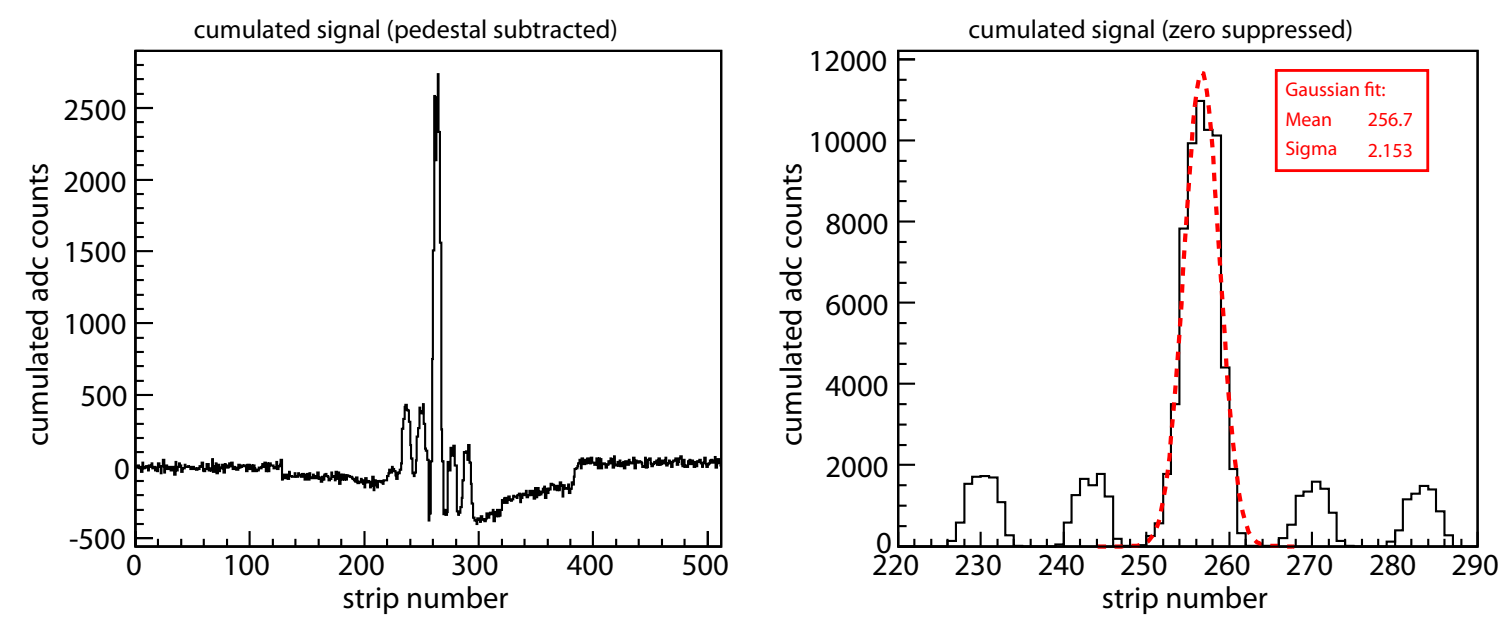

Figure 2: Left - the cumulated signal (2860 events) on a TEC+ ring 4 sensor shows artifacts from illumination with excessive laser amplitude after pedestal subtraction. Right - cumulated signal (2000 events, zero suppressed); the dashed line indicates the best Gaussian fit to the peak of zeroth order. In both cases, additional peaks of the diffraction pattern are visible. The strips have a pitch of $126 \mu \mathrm{m}$.

\subsection{Alignment parameter reconstruction}

The residuals of the reconstructed beam spots with respect to their nominal positions are calculated. Using these residuals, the reconstruction proceeds in a two-fold way: first, the geometry of the large-scale tracker support structure's elements is calculated (standalone reconstruction); second, the reconstructed hit positions are provided as additional input to track-based tracker alignment algorithms. Furthermore, various processed data are passed to the CMS data quality monitoring system.

The standalone reconstruction algorithms are based on an analytical approach of solving the $\chi^{2}$-minimization problem for the laser hit residuals [2]. They are characterized by their fast response and therefore qualify the LAS as an instrument for regular on-time monitoring of the tracker support structure. Two separate algorithms independently calculate the alignment parameters for the end cap internal and the alignment tube systems of beams.

Since the laser beams are fully enclosed within the tracker volume, the coordinate system in which the alignment is performed cannot be related to the CMS coordinate system. Furthermore, it is impossible to disentangle global shearings or twists of the tracker support structure from coherent common displacements of the laser beams. All reconstructed alignment parameters are therefore defined by the constraint that their average value over nine disks (end cap internal beams), or both end caps and the TIB and TOB half-barrels (alignment tube beams), respectively, is zero with respect to the average beam orientation. This results in a non-Cartesian coordinate system which may be twisted and sheared along the $z$-axis. The alignment parameters accessible to the standalone reconstruction are:

- for the end cap internal beam systems - the rotations around the $z$-axis $\left(\Delta \phi_{\mathrm{k}}\right)$ and the translations along $x$ and $y\left(\Delta x_{\mathrm{k}}, \Delta y_{\mathrm{k}}\right)$ of each end cap disk $(k=1, \ldots, 18)$ relative to each other, and 
- for the alignment tube beam system - the translations along $x$ and $y$ and the rotations around all three spatial axes of both end caps and the TIB and TOB half-barrels relative to each other.

For the end caps, the disk parameters $\Delta \phi_{\mathrm{k}}, \Delta x_{\mathrm{k}}$ and $\Delta y_{\mathrm{k}}$ are direct observables by the LAS, while within the alignment tube beam system, the end caps and half-barrels are handled as solid bodies, each described by the positions and rotations of two virtual disks at their respective end faces, which are then handled in a similar way as the TEC disks, and from which the above alignment parameters can be derived.

In addition to the alignment parameters, the laser beams must a priori be assumed to deviate from their nominal orientations. However, thanks to the high redundancy of the LAS design, the deviations of the beams with respect to each other can partly be measured. Two of the four degrees of freedom of a laser beam can be reconstructed (beam parameters). It has been chosen to parameterize the beams by the deviations in $\phi$ of the first and last measured hit position along $z$, respectively $\Delta \phi_{\mathrm{i}}^{-}$and $\Delta \phi_{\mathrm{i}}^{+}$, with $i$ denoting the beam number.

In the end cap internal beam system, the linear residual $\Delta s_{\mathrm{i}, \mathrm{k}}$ on a sensor of TEC disk $k$ in the path of laser beam $i$ is given by

$$
\Delta s_{\mathrm{i}, \mathrm{k}}=-R \cdot \Delta \phi_{\mathrm{k}}+\sin \left(\phi_{\mathrm{i}}\right) \cdot \Delta x_{\mathrm{k}}-\cos \left(\phi_{\mathrm{i}}\right) \cdot \Delta y_{\mathrm{k}}-\left(z_{\mathrm{k}} / L-1\right) \cdot R \cdot \Delta \phi_{\mathrm{i}}^{-}-z_{\mathrm{k}} / L \cdot R \cdot \Delta \phi_{\mathrm{i}}^{+}
$$

with $R$ being the radius of the laser with respect to the beam line, $\phi_{\mathrm{i}}$ its $\phi$ position, $z_{\mathrm{k}}$ the $z$ position of the sensor, and $L$ the end cap length. The spatial symmetries of the beam system design are used to simplify the set of residual equations for the 144 sensors in a TEC. Additionally, all ambiguous coherent displacements of the disks with respect to the beams, such as common movements along $x$ and $y$ as well as common twists and shearings, are absorbed into redundant global parameters. The remaining system of equations can be solved analytically for the disk parameters $\Delta \phi_{\mathrm{k}}, \Delta x_{\mathrm{k}}$ and $\Delta y_{\mathrm{k}}$ as well as the beam parameters $\Delta \phi_{\mathrm{i}}^{-}$and $\Delta \phi_{\mathrm{i}}^{+}$. The reconstruction of the positions and rotations of the end caps and half-barrel subdetectors in the alignment tube system of beams is performed in a similar manner. Finally, the end cap internal disk and beam parameters are transformed into the frame of reference of the alignment tube system of beams.

\section{Results}

Although mainly devoted to commissioning, LAS data from the cosmic muon data taking campaigns were used for the alignment measurements. During the 2008 cosmic muon campaign, 31 out of the 40 laser beams were operational. Fig. 3 compares the alignment parameters $\Delta \phi_{\mathrm{k}}$, $\Delta x_{\mathrm{k}}$ and $\Delta y_{\mathrm{k}}$ as measured in 2008 to the results from the track-based alignment [3], from the LAS sector test and from optical survey measurements [4]. All data are in good agreement. Small discrepancies between the alignment parameters measured in 2008 and the sector test and survey results are expected, since the end caps have been rotated and moved in their final position after the sector test. 

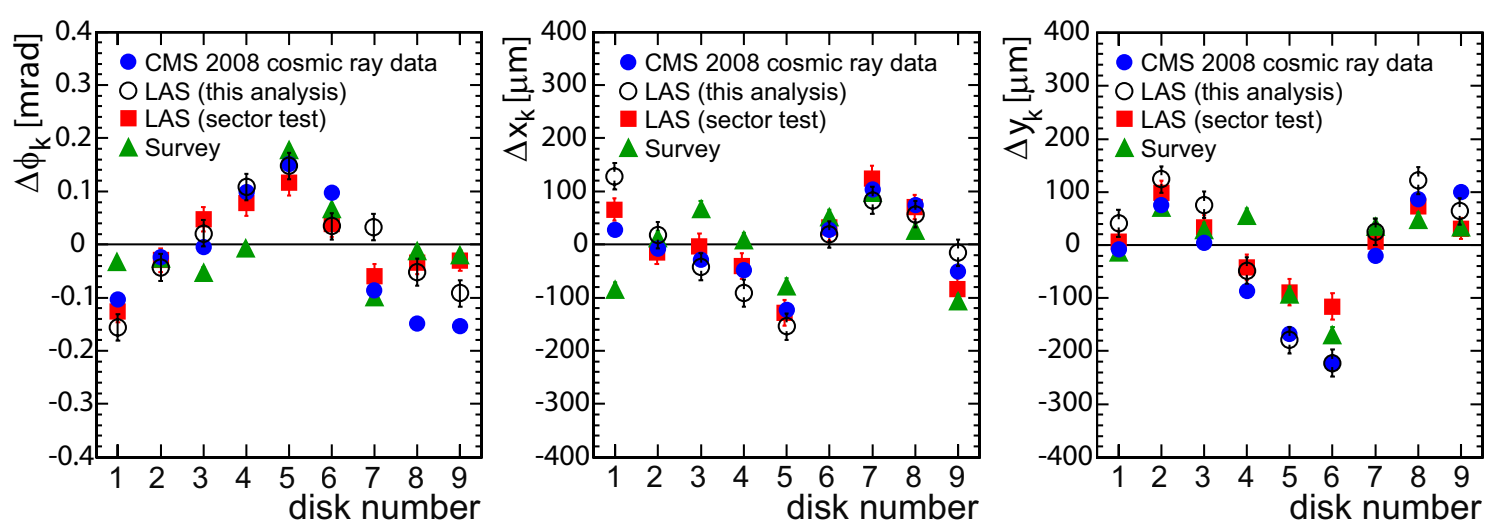

Figure 3: The alignment parameters $\Delta \phi_{\mathrm{k}}, \Delta x_{\mathrm{k}}$ and $\Delta y_{\mathrm{k}}$ for the positive $z$ end cap disks measured by the LAS, by the track-based alignment with cosmic muon data taken in 2008 [3], as well as results from an earlier LAS measurement (sector test) and optical survey [4].

\section{Conclusions}

The Laser Alignment System of the CMS Silicon Strip Tracker provides a fast inter-alignment of the TEC disks as well as of the TIB, TOB and TEC subdetectors with respect to each other. With the data taken during the CMS cosmic muon campaigns, the system is fully commissioned and operational.

Results from the forward tracker end cap, which is currently the best studied and understood CMS subdetector in terms of LAS operation, show that the alignment parameters measured with the LAS are in good agreement with those obtained with track-based alignment algorithms. Furthermore, the distribution of residuals between the datasets confirms that the LAS is able to measure the geometry of the large-scale support structure of the tracker with an absolute precision better than $100 \mu \mathrm{m}$.

\section{References}

[1] CMS Collaboration, JINST 3 (2008) S08004

[2] B. Wittmer, PhD Thesis, RWTH Aachen (2002), http://darwin.bth.rwth-aachen.de/opus3/volltexte/2002/348

[3] CMS Collaboration, arXiv:0910.2505 (accepted by JINST)

[4] CMS Tracker Collaboration, JINST 4 (2009) T07001 